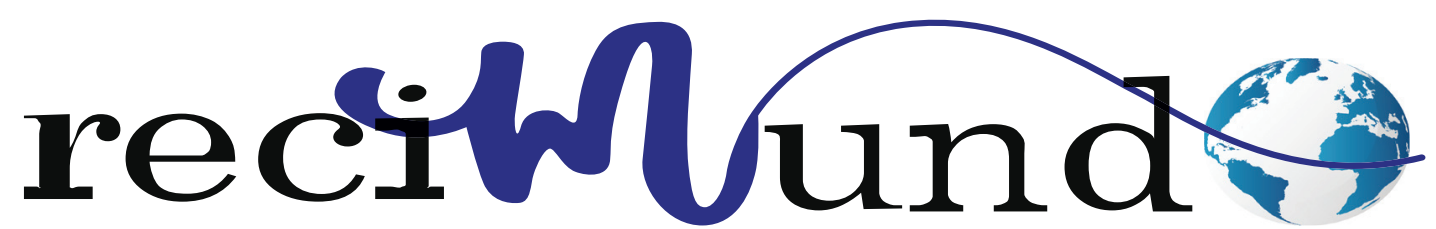

Revista Científica Mundo de la Investigación y el Conocimiento

DOI: 10.26820/recimundo/4.(1).enero.2020.26-38

URL: http://recimundo.com/index.php/es/article/view/730

EDITORIAL: Saberes del Conocimiento

REVISTA: RECIMUNDO

ISSN: 2588-073X

TIPO DE INVESTIGACIÓN: Artículo de Revisión

CÓDIGO UNESCO: 3205 Medicina Interna

PAGINAS: 26-38

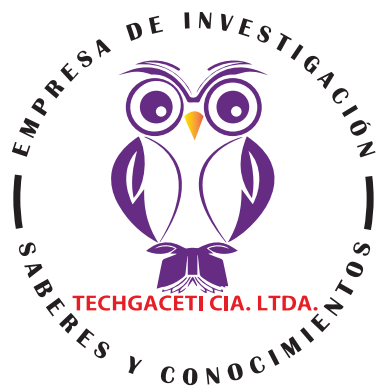

\title{
Alternativas a la intubación orotraqueal ante una vía aérea difícil
}

\section{Alternatives to orotracheal intubation before a difficult airway}

Alternativas à intubação orotraqueal antes de uma via aérea

Daysi Katrina Ronquillo Guachamin; Edgar Lizandro Quezada Miranda2; Sabrina Esther Saltos Moran³;

Sonia Maria González Mora ${ }^{4}$

RECIBIDO: 18/12/2018 ACEPTADO: 29/03/2019 PUBLICADO: 01/12/2019

1. Médico; Investigador Independiente; Guayaquil, Ecuador; katrina.ronqui@gmail.com; (DD https://orcid. org/0000-0003-2552-8920

2. Médico; Investigador Independiente; Guayaquil, Ecuador; edgarqur6d@gmail.com; (D) https://orcid.org/00000002-7121-5629

3. Médico; Investigador Independiente; Guayaquil, Ecuador; sabrinasaltosm@hotmail.com; (D) https://orcid. org/0000-0002-8134-4328

4. Médico; Investigador Independiente; Guayaquil, Ecuador; pingui_smgm@hotmail.com; (D) https://orcid.org/0000-0002-6241-428X

\section{CORRESPONDENCIA}

Daysi Katrina Ronquillo Guachamin

katrina.ronqui@gmail.com

Guayaqui, Ecuador 


\section{RESUMEN}

La vía aérea por definición es un conducto por el cual pasa el aire; o bien, es la ruta por la cual transita el aire desde la nariz o la boca hacia los pulmones. De tal forma, la vía aérea difícil puede definirse como la complejidad en el acceso del conducto por el cual pasa el aire desde la nariz o la boca hacia los pulmones. Según la guía de recomendación de la American Society of Anesthesiologists (ASA), ésta se define como una situación clínica en la cual un anestesiólogo con entrenamiento convencional experimenta dificultad para la ventilación de la vía aérea superior con una mascarilla facial, dificultad para la intubación traqueal, o ambas. En tal sentido, se logra establecer que, la vía aérea difícil (VAD) representa una interacción compleja entre factores del paciente, el entorno clínico, las habilidades y preferencias del realizador, quien se enfrenta a las siguientes dificultades en el abordaje: (1) dificultad para la ventilación con mascarilla facial, (2) dificultad en la realización de la laringoscopía convencional después de múltiples intentos, (3) dificultad durante la intubación traqueal después de múltiples intentos en presencia o ausencia de patología traqueal y (4) intubación fallida La dificultad para la intubación se presenta cuando se han realizado más de tres intentos de intubación utilizando la laringoscopía convencional en condiciones óptimas y por personal experimentado. El control de la vía aérea en el paciente crítico puede resultar a menudo difícil por diversas razones: pre-oxigenación ineficiente, el carácter urgente de la situación, las condiciones fisiológicas adversas del paciente crítico, las comorbilidades asociadas y/o la condición de estómago lleno de la mayoría de estos pacientes. Los signos de una inadecuada ventilación son: ausencia de ruidos respiratorios, obstrucción severa e insuflación gástrica en la auscultación, cianosis, inadecuados valores de saturación arterial de oxigeno (Sa02).

Palabras clave: Alternativas de Intubación Orotraqueal; Vía Aérea Difícil; Laringoscopia; Patología Traqueal; Intubación Fallida; Laringoscopia Convencional.

\section{ABSTRACT}

The airway by definition is a conduit through which the air passes; or, it is the route by which the air travels from the nose or mouth to the lungs. In this way, the difficult airway can be defined as the complexity in the access of the duct through which air passes from the nose or mouth to the lungs. According to the recommendation guide of the American Society of Anesthesiologists (ASA), this is defined as a clinical situation in which an anesthesiologist with conventional training experiences difficulty in the ventilation of the upper airway with a facial mask, difficulty in tracheal intubation, or both. In this sense, it is possible to establish that, the difficult airway (VAD) represents a complex interaction between patient factors, the clinical environment, the skills and preferences of the filmmaker, who faces the following difficulties in the approach: (1) difficulty for ventilation with a face mask, (2) difficulty in performing conventional laryngoscopy after multiple attempts, (3) difficulty during tracheal intubation after multiple attempts in the presence or absence of tracheal pathology and (4) failed intubation Intubation difficulty arises when more than three intubation attempts have been made using conventional laryngoscopy under optimal conditions and by experienced personnel. The control of the airway in the critical patient can often be difficult for several reasons: inefficient pre-oxygenation, the urgent nature of the situation, the adverse physiological conditions of the critical patient, the associated comorbidities and / or the condition of a full stomach of most of these patients. Signs of inadequate ventilation are: absence of respiratory noises, severe obstruction and gastric insufflation in auscultation, cyanosis, inadequate values of arterial oxygen saturation (SaO2).

Keywords: Orotracheal Intubation Alternatives; Difficult Airway; Laryngoscopy; Tracheal Pathology; Failed intubation; Conventional laryngoscopy.

\section{RESUMO}

A via aérea, por definição, é um canal pelo qual o ar passa; ou, é a rota pela qual o ar viaja do nariz ou da boca para os pulmões. Dessa maneira, a via aérea difícil pode ser definida como a complexidade no acesso do duto através do qual o ar passa do nariz ou da boca para os pulmões. De acordo com o guia de recomendação da Sociedade Americana de Anestesiologistas (ASA), isso é definido como uma situação clínica em que um anestesiologista com treinamento convencional experimenta dificuldade na ventilação das vias aéreas superiores com máscara facial, dificuldade na intubação traqueal ou ambas. Nesse sentido, é possível estabelecer que a via aérea difícil (DAV) representa uma interação complexa entre os fatores do paciente, o ambiente clínico, as habilidades e preferências do cineasta, que enfrenta as seguintes dificuldades na abordagem: (1) dificuldade para ventilação com máscara facial, (2) dificuldade em realizar laringoscopia convencional após várias tentativas, (3) dificuldade durante a intubação traqueal após várias tentativas na presença ou ausência de patologia traqueal e (4) falha na intubação quando ocorre mais de três foram feitas tentativas de intubação usando laringoscopia convencional em condições ideais e por pessoal experiente. O controle das vias aéreas no paciente crítico muitas vezes pode ser difícil por várias razões: pré-oxigenação ineficiente, natureza urgente da situação, condições fisiológicas adversas do paciente crítico, as comorbidades associadas e / ou a condição de estômago cheio da maioria desses pacientes. Os sinais de ventilação inadequada são: ausência de ruídos respiratórios, obstrução grave e insuflação gástrica na ausculta, cianose, valores inadequados de saturação arterial de oxigênio (Sa02).

Palavras-chave: Alternativas de intubação orotraqueal; Via aérea difícil; Laringoscopia; Patologia Traqueal; Falha na intubação; Laringoscopia convencional. 


\section{Introducción}

El control de la vía aérea (VA) en quirófano es una responsabilidad del anestesiólogo, es por ello que este especialista al finalizar su formación debe haber desarrollado las habilidades necesarias para atender esta exigencia. El control adecuado de la vía aérea (VA) para garantizar una correcta ventilación y oxigenación no sólo es indispensable en muchos procedimientos anestésico-quirúrgicos sino también en todas aquellas situaciones en las que la función respiratoria está comprometida. No obstante, en los últimos años se ha producido una proliferación de nuevos dispositivos y se han mejorado las técnicas de intubación y ventilación proporcionando una gran ayuda en el abordaje de la vía aérea difícil (VAD). (La Salle, 2016)

En virtud de esto, el manejo óptimo de la vía aérea y ventilación de pacientes críticos sigue siendo un pilar básico en la supervivencia, evolución y pronóstico; la intubación orotraqueal (IOT) es el gold standard en estas situaciones. (La Salle, 2016). De allí, que conocer la posición, las maniobras y el material facilitador de la IOT, así como realizar una oxigenación-ventilación previa y una sedorrelajación adecuadas, aumentará de forma significativa las posibilidades de éxito. Por ende, es importante comprender que, la primera causa de dificultad para manejar una vía aérea no la ofrece el paciente, sino el personal que la maneja, y se vuelve crítico cuando se enfrenta a una persona con alteraciones anatómicas como trauma maxilofacial, obesidad mórbida, artritis reumatoide, alteraciones congénitas, entre otras.

Los médicos y el personal paramédico, con buena formación y entrenamiento para resolver una vía aérea difícil, harán que los dispositivos supraglóticos tengan el valor adecuado en el momento de una emergencia. La extubación está asociada con dificultades en la reintubación en 2/3 de los pacientes. (Malaponte, 2016). La permea- bilización de la vía aérea es el aspecto más preocupante para cualquier médico cuando se enfrenta a un paciente con trauma de columna cervical; la mayor parte de los movimientos de la columna cervical en las maniobras de permeabilización de la vía aérea incluye las articulaciones atlantooccipital y atlantoaxoidea.

De allí, que la máscara laríngea ha sido utilizada con éxito para lograr intubación de pacientes con compromiso de la columna cervical. Investigaciones recientes demuestran que la máscara laríngea produce mínimos movimientos cervicales en flexión, contrario a lo que ocurre con las maniobras de laringoscopia, situación que es beneficiosa en pacientes con compromiso de estructuras que producen inestabilidad en extensión. (Malaponte, 2016).

Los pacientes con lesiones cervicales altas presentan una capacidad residual funcional respiratoria disminuida, provocando alteraciones en la ventilación y oxigenación aun cuando se inicie en poco tiempo y se preoxigene previamente al paciente. (Malaponte, 2016). Cualquier dispositivo supraglótico que logre disminuir estos períodos constituye una alternativa a considerar. Entiéndase entonces que, las modalidades validadas en otras condiciones como alternativas a la intubación orotraqueal, como los dispositivos supraglóticos, demuestran ser de suma utilidad ante todo paciente grave que requiere ventilación ó protección de la vía aérea.

Por tal razón, en todo servicio de emergencias debe existir, en el carro de reanimación, el material para intubación difícil, con el mismo material que los carros de las áreas quirúrgicas, debiendo ser chequeados de forma regular, reemplazando el material si esto fuera necesario. Dichos carros de reanimación deben contar con: laringoscopio rígido con palas de Miller y Macintosh de distintos tamaños, fiador semirígido (Eschman), alternativa a la máscara facial para la ventilación difícil o imposible (mascarilla 
laríngea, combitube), lo cual constituye una alternativa a la laringoscopia directa para la intubación fallida (Fastrach + tubo endotraqueal para Fastrach), equipo para acceso quirúrgico urgente de la vía aérea (cricotirotomía, traqueotomía), tubos endotraqueales y cánulas orofaringeas de distintos calibres (acorde a las necesidades potenciales según tipo de pacientes), entre los más importantes. (Malaponte, 2016).

Las ventajas de estos dispositivos frente al tubo endotraqueal convencional se pueden resumir en: son mínimamente invasivos, producen cambios mínimos a nivel pulmonar y en la fisiología cardiovascular, menos resistencia al flujo de aire, se reduce la incidencia de laringoespasmo, el período de recuperación postanestésica es menor y se tiene un manejo del CO2 similar con menos agresión. (Malaponte, 2016).Además presentan otras ventajas como: un alto porcentaje de colocación correcta en un primer intento, disminución en los tiempos requeridos para lograr restablecer la ventilación, disminución de complicaciones deducibles del abordaje de la vía aérea (lesiones odontológicas, de la mucosa de la cavidad oral, la lengua, la epiglotis entre otras), sangrados, entre los más significativos, lo que es posible aun en manos expertas cuando se aborda la vía aérea difícil.

\section{Fuentes documentales}

El análisis correspondiente a las características que predomina en el tema seleccionado, llevan a incluir diferentes fuentes documentales encargadas de darle el respectivo apoyo y en ese sentido cumplir con la valoración de los hechos a fin de generar nuevos criterios que sirven de referencia a otros procesos investigativos. Para (Arias, 2010), las fuentes documentales incorporadas en la investigación documental o bibliográfica, "representa la suma de materiales sistemáticos que son revisados en forma rigurosa y profunda para llegar a un análisis del fenómeno". (p.41). Por lo tanto, se procedió a cumplir con la realización de una lectura previa determinada por encontrar aquellos aspectos estrechamente vinculados con el "ALTERNATIVAS A LA INTUBACIÓN OROTRAQUEAL ANTE UNA VÍA AÉREA DIFÍCIL" para luego explicar mediante un desarrollo las respectivas apreciaciones generales de importancia.

\section{Técnicas para la Recolección de la Infor- mación}

La conducción de la investigación para ser realizada en función a las particularidades que determinan a los estudios documentales, tiene como fin el desarrollo de un conjunto de acciones encargadas de llevar a la selección de técnicas estrechamente vinculadas con las características del estudio. En tal sentido, (Arias Ob cit) refiere, que es "una técnica particular para aportar ayuda a los procedimientos de selección de las ideas primarias y secundarias". (p. 71).

Por ello, se procedió a la utilización del subrayado, resúmenes, fichaje, como parte básica para la revisión y selección de los documentos que presentan el contenido teórico. Es decir, que mediante su aplicación de estas técnicas se pudo llegar a recoger informaciones en cuanto a la revisión bibliográfica de los diversos elementos encargados de orientar el proceso de investigación. Tal como lo expresa, (Arias Ob cit) "las técnicas documentales proporcionan las herramientas esenciales y determinantes para responder a los objetivos formulados y llegar a resultados efectivos" (p. 58). Es decir, para responder con eficiencia a las necesidades investigativas, se introdujeron como técnica de recolección el método inductivo, que hizo posible llevar a cabo una valoración de los hechos de forma particular para llegar a la explicación desde una visión general.

Asimismo, se emplearon las técnicas de análisis de información para la realización de la investigación que fue ejecutada bajo la dinámica de aplicar diversos elementos encargados de determinar el camino a recorrer por el estudio, según, (Arias, Ob cit)

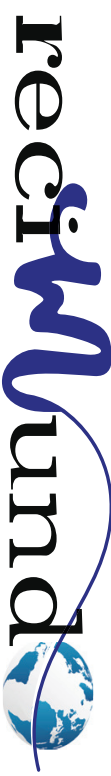


las técnicas de procesamiento de datos en los estudios documentales "son las encargadas de ofrecer al investigador la visión o pasos que debe cumplir durante su ejercicio, cada una de ellas debe estar en correspondencia con el nivel a emplear" ( $p$. 123). Esto indica, que para llevar a cabo el procesamiento de los datos obtenidos, es necesario establecer las técnicas que serán seleccionadas, destacándose en este caso, de manera particular: fichas de resumen, textual, registros descriptivos entre otros, los mismos se deben ajustar al nivel que ha sido seleccionado.

\section{Resultados}

\section{Vía aérea difícil}

Según la Sociedad Americana de Anestesiología (SAA), se define la vía aérea difícil como la situación clínica en la que personal entrenado y experimentado encuentra impedimentos para conseguir la ventilación mediante mascarilla facial, para la intubación traqueal o para ambas técnicas. (Garza, 2015) Esta situación viene dada por la interacción de distintos factores: el paciente, el entorno clínico y la capacidad de quien realiza la técnica. Se han propuesto otras definiciones en relación con la vía aérea:

\section{Dificultad para la ventilación con mas- carilla facial o dispositivo supraglótico:}

- No es posible ventilar por uno o varios de los siguientes problemas: sellado inadecuado de la mascarilla, excesiva pérdida de gas, o excesiva resistencia para introducir o expulsar el gas.

- Signos de ventilación con mascarilla inadecuada son: ausencia de movimientos torácicos, ausencia de ruidos respiratorios, signos de obstrucción severa a la auscultación, cianosis, distensión gástrica, descenso de la saturación de oxígeno, ausencia o medida inadecuada de dióxido de carbono exhalado, y cambios hemodinámicos asociados con hipoxemia o hipercapnia (ej. hiperten- sión, taquicardia, arritmias,...). (Garza, 2015)

\section{Dificultad para la implantación de dis-} positivo supraglótico: se requieren múltiples intentos para la colocación de un dispositivo supraglótico, en presencia o ausencia de patología traqueal.

3. Laringoscopia difícil: cuando no es posible visualizar ninguna porción de las cuerdas vocales tras múltiples intentos de laringoscopia convencional.

\section{Intubación traqueal difícil: El enfermo crítico}

- Cuando la intubación traqueal requiere múltiples intentos haya o no patología traqueal.

- Necesidad de más de 3 intentos de intubación o cuando los intentos de intubación duran >10 minutos.

5 . Intubación fallida: no se consigue la colocación del tubo traqueal tras múltiples intentos.

6. Vía aérea quirúrgica transtraqueal difícil: aquella que requiere un tiempo excesivo o múltiples intentos para su realización.

El algoritmo de vía aérea difícil de la American Society of Anesthesiologist propone intubación con el paciente despierto como alternativa ante una previsible vía aérea difícil. En este caso el paciente despierto se intubó con el apoyo de videolaringoscopio. Los videolaringoscopios son dispositivos que surgen ante la inquietud de asegurar la vía aérea en aquellas personas donde se reconozca una vía aérea difícil, proporcionan una visión aumentada y mejorada de la glotis y, sin necesidad de alinear los ejes, se obtiene mejoría en al menos un grado en la escala de Cormack-Lehane comparados con lalaringoscopia convencional; así como riesgo disminuido de traumatismo de la vía aérea. Son más sencillos de utilizar que un fibroscopio y tienen el mismo diseño que un laringoscopio convencional. De allí, que la 
Cuadro 1. Entidades Clínicas asociadas a una vía difícil

\begin{tabular}{|l|}
\hline \multicolumn{1}{|c|}{ Entidades clínicas asociadas a una vía aérea difícil } \\
\hline Intubaciones Previas \\
\hline Enfermedades Respiratorias \\
\hline Problemas Dentarios \\
\hline Artritis Reumatoide \\
\hline Coagulopatías \\
\hline Síndromes Congénitos \\
\hline Diabetes Mellitus \\
\hline Patologías Obvias \\
\hline Obesidad \\
\hline
\end{tabular}

Fuente: Parra, 2017

Cuadro 2. Escala para la valoración de la apertura de la boca tomando los incisivos superiores e inferiores como referencia

Valoración de apertura de la boca tomando incisivos como valor de referencia

\begin{tabular}{|ll|}
\hline Clase I & Más de $3 \mathrm{~cm}$ \\
\hline Clase II & 2.6 a $3 \mathrm{~cm}$ \\
\hline Clase III & De 2 a $2.5 \mathrm{~cm}$ \\
\hline Clase IV & Menos de $2 \mathrm{~cm}$ \\
\hline
\end{tabular}

Fuente: Parra, 2017

experiencia del anestesiólogo es un factor que no puede ser subestimado. (Garza, 2015)

\section{Manejo de la vía aérea difícil vad}

La literatura sugiere que el empleo de estrategias específicas facilita la intubación de la VAD. Pese a que el grado de beneficio de cualquier estrategia específica no ha sido determinado, el consenso entre los expertos apunta hacia que la elaboración de un plan para el manejo de esta entidad garantiza un mejor resultado final. (Parra, 2017). Debido a que la VAD representa una entidad que pone potencialmente en peligro la vida, la elaboración de algoritmos para su manejo proporciona una estrategia que ayuda a la resolución de eventualidades adversas, las cuales dependen en cierta forma de la condición del paciente, de las habilidades y preferencias del realizador. Por tal razón, el anestesiólogo que se enfrenta a una probable VAD debe cuestionarse lo siguiente:

(1) ¿se requiere del manejo de la vía aérea?

(2) ¿la laringoscopía puede ser difícil de realizar?

(3) ¿la ventilación supralaríngea puede ser difícil de realizar?

(4) ¿existe el riesgo de aspiración?

He allí, que las respuestas a estas preguntas críticas pueden proporcionar una evaluación más completa de cualquier vía aérea; o bien, pueden servir para la elaboración de esfuerzos enfocados al manejo adecuado de la misma. (Parra, 2017). El algoritmo propuesto por la ASA propone que la evaluación anticipada y probable del impacto clínico puede prever problemas básicos que pueden presentarse de forma aislada o combinada durante el manejo de la vía aérea. No obstante, no existen contraindicaciones absolutas para este procedimiento, salvo una alergia verdadera a los anestésicos locales. Para asegurar el éxito de la intubación con el paciente despierto, es esencial la preparación adecuada del paciente consiste en:

- Proporcionar al paciente información sobre la realización del procedimiento, así

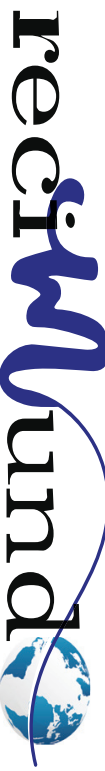




\section{como brindarle apoyo psicológico}

- La utilización de agentes que sequen las mucosas como el glicopirrolato $0.2 \mathrm{mg}$. IV

- Proporcionar una sedación que permita la cooperación del paciente, esto se puede llevar a cabo con la utilización de narcóticos como el fentanilo a dosis que permitan este efecto

- Vasoconstricción de mucosas

- La aplicación de anestésicos locales tópicos

- El bloqueo de nervios por inyección como el bloqueo del nervio glosofaríngeo y el bloqueo del nervio laríngeo superior

- Preoxigenación

- La profilaxis de la aspiración de contenido gástrico. Una vez que el paciente ha sido preparado la intubación se puede realizar por medio de varias técnicas las cuales dependen del entrenamiento, habilidades y preferencias del realizado. (Parra, 2017)

Bajo estas observaciones se establece que, entre las recomendaciones a seguir para tomar una vía aérea se recomienda realizar una historia clínica. La intención de realizar una historia clínica de la vía aérea es el detectar factores anestésicos, médicos o quirúrgicos que puedan indicar la presencia de VAD. El examinar registros anestésicos previos siempre que éstos existan, pueden proveernos de información útil. Existe una asociación demostrada entre la VAD y enfermedades congénitas, adquiridas o eventos traumáticos. (Parra, 2017). Según el autor, estas entidades se pueden dividir en dos grupos:

- El grupo I son aquellas entidades con datos evidentes de VAD, por ejemplo: tumores, abscesos o deformidades congénitas.
- El grupo II son aquellas entidades sin signos evidentes de VAD.

Del mismo modo, se plantea la exploración física la cual no ha podido medirse clínicamente, no obstante, hay suficiente información que datos obtenidos en la exploración física pueden estar relacionados con la presencia de VAD. La realización de la exploración física de la vía aérea resulta en facilitar la detección de una VAD. (Parra, 2017). Por consiguiente, no existe un solo indicador previo al procedimiento que determine dificultad a la ventilación, laringoscopía o intubación. De la misma forma ningún examen es determinante e infalible, la predicción de la VAD durante la realización de la exploración física requerirá de un mayor número de exámenes durante la misma.

Hallazgos específicos de la exploración física de la vía aérea han sido incorporados en numerosos sistemas de evaluación CON con la intención de predecir una VAD. Los sistemas de evaluación que existen para la predicción de VAD han mostrado una modesta especificidad y sensibilidad. (Barreto, 2017). En virtud de esto, se logra señalar que la combinación de sistemas de evaluación han demostrado mayor sensibilidad. La exploración física de la vía aérea no requiere de equipo especial y no toma más que un minuto, primero se enfoca a los dientes (apertura de la boca, tamaño de los dientes) posteriormente a la exploración del interior de la boca (escala de Mallampati) continúa con el espacio mandibular (grado de protrusión mandibular, valoración de la curvatura del paladar) y finalmente con el cuello (distancia tiromentoniana y rango de la movilidad de la cabeza y cuello). De tal manera que al valorar la vía aérea se debe tomar en cuenta:

- El tamaño de la lengua,

- La movilidad del cuello y

- Proximidad de la laringe a la base de la lengua; estos elementos modificarán la alineación de los ejes de la vía aérea 
para la correcta visualización del orificio

glótico. (Barreto, 2017).

Cuadro 3. Escala para la valoración de protrusión mandibular

\section{Valoración de Protusión Mandibular}

Clase I. Los incisivos inferiores pueden ser llevados más adelante de la arcada dental superior

Clase II. Los incisivos inferiores se deslizan hasta el nivel de la dentadura superior, es decir, quedan a la misma altura $1 / 3$

Clase III. Los incisivos inferiores no se proyectan hacia adelante y no pueden tocar la arcada dentaria superior

Fuente: Barreto, 2017

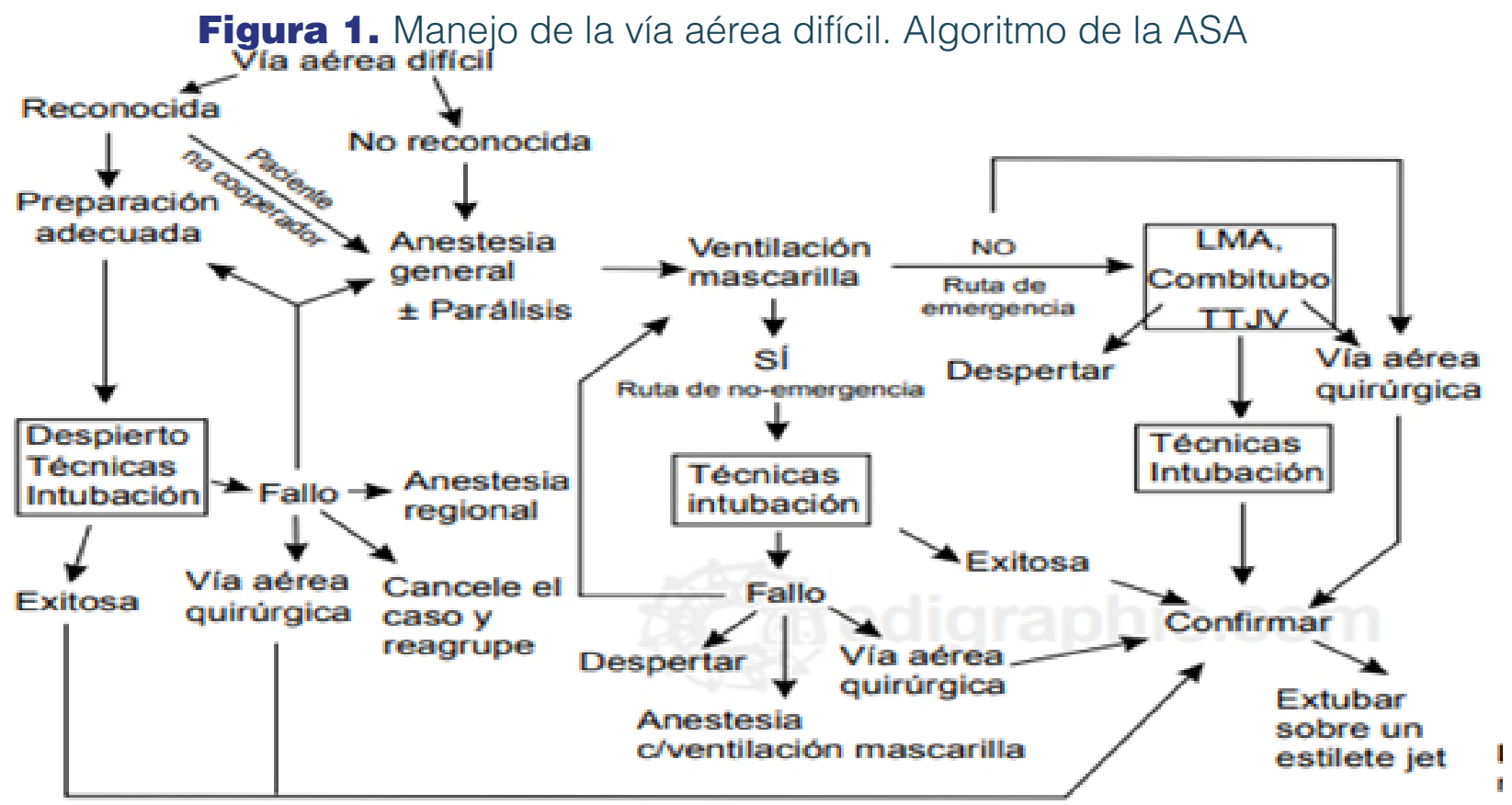

Fuente: Barreto, 2017

La ventilación, y por tanto la permeabilización y el aislamiento de la vía aérea (VA) en el paciente portador de una emergencia vital, es uno de los retos a los que cotidianamente se deben enfrentar los médicos y enfermeros dedicados a las urgencias y emergencias tanto pre hospitalarias como hospitalarias. Este apoyo a la ventilación se ha venido realizando mediante dos procedimientos principalmente, uno no invasivo como es la ventilación con presión positiva mediante mascarilla facial y balón de resucitación auto hinchable, y otro invasivo como es la intubación naso u orotraqueal (IOT) mediante laringoscopia directa. (Barreto, 2017).

Asimismo, se han desarrollado multitud de programas formativos y cursos en los que se han formado tanto en teoría como en práctica a los profesionales más relacionados con los pacientes candidatos de estas técnicas (Urgencias de Atención Primaria de Salud [APS], sistemas de Emergencia, Servicios de Urgencias Hospitalarios, etc.). Pero tras una adecuada formación teóri- 
co-práctica, es la actividad diaria y la realización frecuente de estas técnicas lo que permite mantener y alcanzar un alto grado de experiencia y destreza en las mismas. (Barreto, 2017).

Igualmente, existen situaciones en las que estas técnicas no son fácilmente aplicables, debido al contexto donde deben ser realizados, así como a las alteraciones anatómicas y patológicas que presenta el paciente, encontrándonos con relativa frecuencia ante pacientes en los que la IOT es complicada o imposible, debiendo ser mantenidos ventilatoriamente mediante ventilación con balón resucitador. La situación se complica aún más cuando, ante la imposibilidad de aislar la VA mediante IOT, somos incapaces de mantener una saturación de oxígeno (SatO2) > 90\% mediante la ventilación con balón resucitador + bolsa reservorio conectada a oxígeno al 100\% (15 litros de flujo). Esta situación de no intubación y no ventilación con balón resucitador es lo que define una VA difícil (VAD). (Barreto, 2017).

Tal y como se ha indicado anteriormente, la ventilación con balón resucitador y la IOT son los pilares fundamentales en el manejo de la VA de pacientes con fallo para mantener una adecuada permeabilidad de la misma y una óptima ventilación. Dependiendo de la dificultad que encontremos en la aplicación de estas técnicas podemos hablar de:

- Ventilación con mascarilla facial difícil: incapacidad de mantener una SatO2 superior al 90\% aplicando oxígeno al $100 \%$ un reanimador solo.

- Laringoscopia difícil: cuando no es posible visualizar ninguna porción de las cuerdas vocales mediante laringoscopia directa. Gracias a la aplicación de diferentes manio-bras se puede mejorar esta visualización o bien intentar IOT a ciegas.

- Intubación traqueal difícil: necesidad de más de tres intentos para realizar intu- bación traqueal ante una laringoscopia no difícil.

- VAD: es aquella situación clínica en la que nos encontramos ante una ventilación con mascarilla facial difícil más una intubación traqueal difícil, es decir, en la que personal experimentado en manejo de la VA tiene dificultad para mantener una SatO2 del 90\% ventilando con ambú conectado a oxígeno al 100\% y dificultad para la IOT tras tres intentos consecutivos. (Barreto, 2017).

Aunque no es el objetivo del presente artículo, queremos realizar unos breves comentarios sobre las medidas y técnicas que se han venido desarrollando con la finalidad de poder hacer frente a las situaciones antes definidas. En multitud de ocasiones la asistencia de un colaborador en el desarrollo de la técnica, o aplicando maniobras coadyuvantes, va a permitir la ventilación del paciente, superando con ello la tan temida situación de VAD. En otros casos es el uso de guías flexibles y un adecuado alineamiento de los ejes oro-faringo-laríngeos lo que va a permitir hacer frente a cada una de estas eventualidades.

\section{Intubación orotraqueal ante una vía aérea difícil}

Los pasos de la preparación y realización de la técnica de intubación orotraqueal a ciegas con guía metálica atraumática son:

Preparación y selección del tubo: El tamaño del tubo orotraqueal debe seleccionarse de forma adecuada para cada paciente, dependiendo del sexo y talla del mismo. Esto con el fin de lograr pasar a través de las cuerdas vocales y su fácil deslizamiento por la traquea. Universalmente, los tamaños aceptados para la selección del tubo orotraqueal son 7.0 - 7.5 para mujeres y 7.5 8.0 para hombres. Como en cualquier caso de intubación traqueal, debe existir disponibilidad del equipo de vía aérea, previendo que se pueda llegar a presentar alguna eventualidad o la necesidad de usar otros 
dispositivos para ventilación e intubación. (Barreto, 2017).

El direccionamiento del tubo orotraqueal está dado básicamente por la colocación de un inductor o guía metálica recubierta, flexible, atraumática, la cual debe permitir una angulación en el extremo distal del tubo y al mismo tiempo facilitar la acomodación, sin llegar a generar cambios de la forma preestablecida. Se debe dejar la punta de la guía metálica flexible atraumática a nivel de la punta del tubo sin sobresalir el orificio distal, y de esta manera evitar la posibilidad de lesión de tejidos blandos. Para la realización de la intubación orotraqueal a ciegas se recomienda una lubricación interna adecuada del tubo orotraqueal con lidocaína spray, para facilitar el deslizamiento y retiro de la guía metálica cuando se ingrese el tubo a la traquea.

Imagen 1. Tubo Orotraqueal

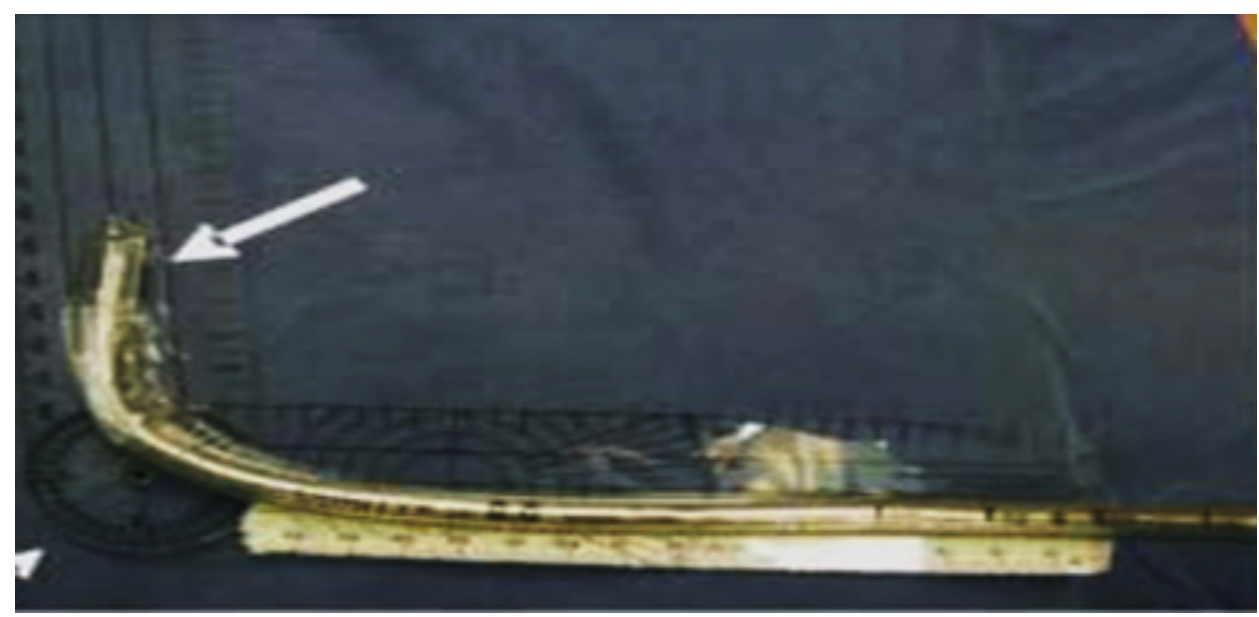

Fuente: Barreto, 2017

Imagen 2. Preparación del paciente para Intubación

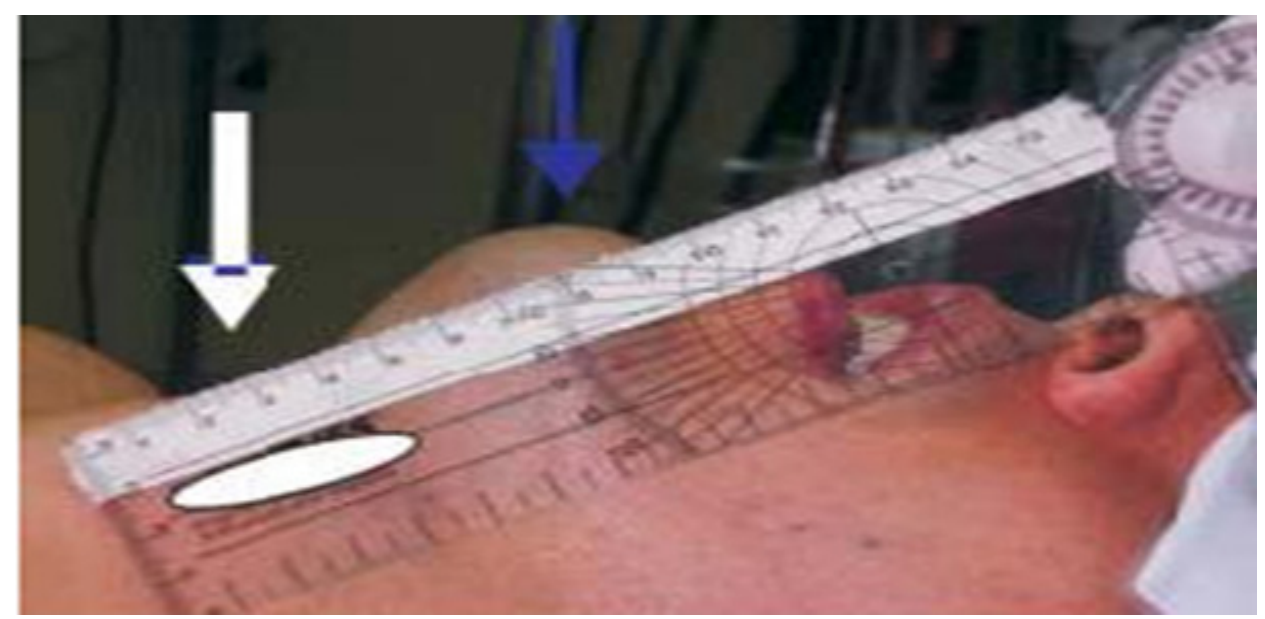

Fuente: Barreto, 2017 
Imagen 3. Introducción del Tubo

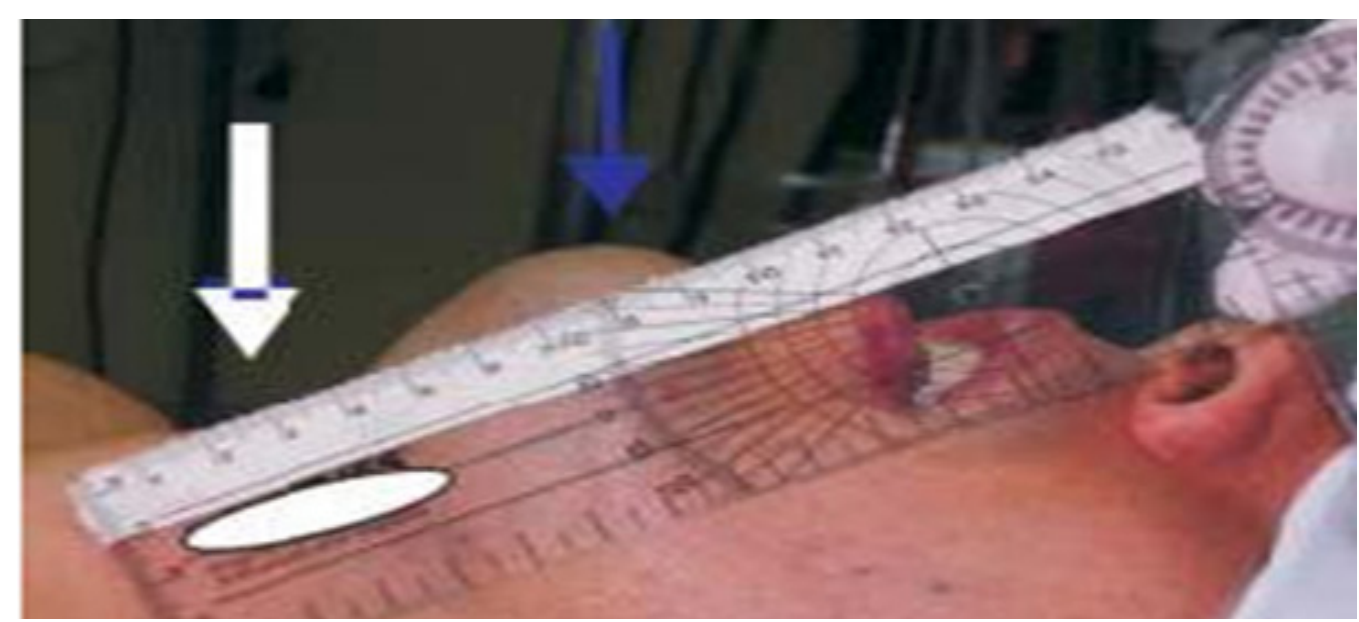

Fuente: Barreto, 2017

4. Con la mano izquierda, sin haber perdido la fijación de la mandíbula, se debe realizar una tracción hacia arriba.

5. Con la mano derecha se ubica el tubo en la línea media, inmediatamente por encima del cartílago tiroides. En ese momento, si la ubicación es adecuada, el tubo se podrá introducir $1-2 \mathrm{~cm}$, sin desviarse de la línea media y sin evidenciarse algún obstáculo para su avance. Como resultado de la progresión alcanzada, se observa un relieve o levantamiento a nivel del cartílago cricoides o inmediatamente por debajo de éste, generado por el extremo biselado del tubo. Esto último es consecuencia del levantamiento de la laringe cuando el tubo se encuentra adecuadamente direccionado. (Vieira, 2017)

\section{Imagen 4.Intubación Orotraqueal}

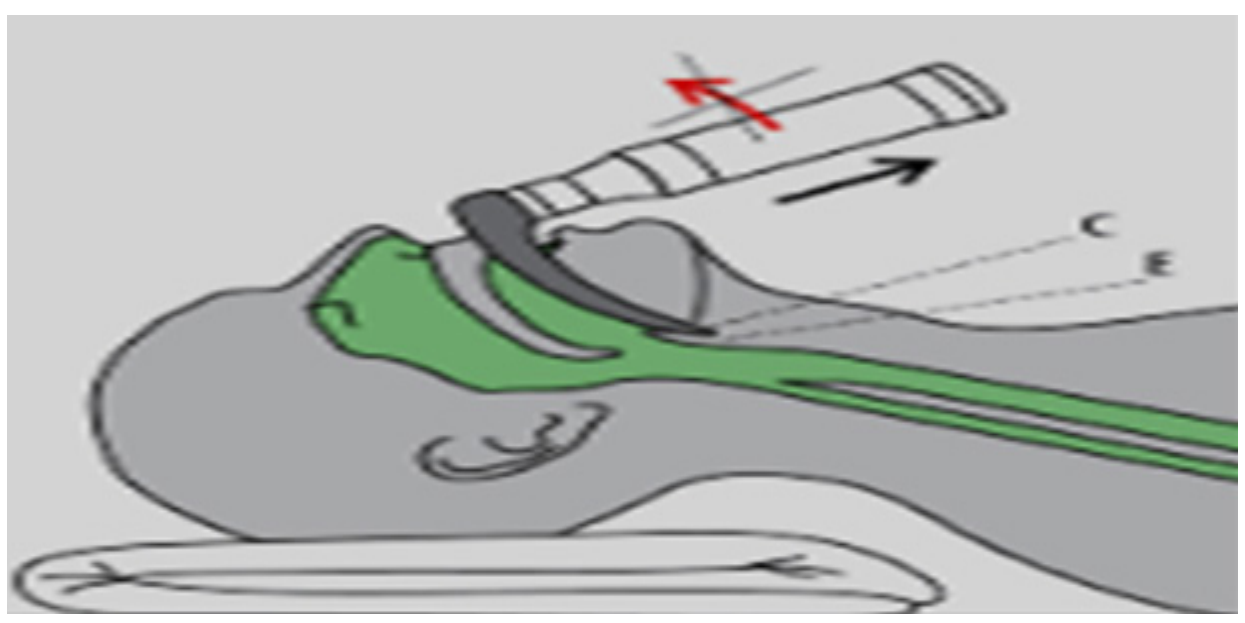

Fuente: Barreto, 2017 
6. En ese momento el tubo orotraqueal será avanzado suavemente 2 a $3 \mathrm{~cm}$ en dirección caudal, sin progresar la guía. La ubicación correcta se confirmará por medio de un movimiento de rotación lateral derecho e izquierdo. Una vez colocado el tubo en la traquea, no será posible desplazarlo lateralmente, tomando esto como parámetro indirecto para confirmación de intubación.

7. Se libera la fijación mandibular y con la mano izquierda se desliza el tubo orotraqueal, mientras con la mano derecha se mantiene fija la guía metálica. Si se presenta limitación u obstrucción para la introducción, se debe realizar una rotación suave en dirección contraria a las manecillas de reloj, para facilitar el ingreso del tubo a la traquea.

8. Al momento en que se realiza el deslizamiento del tubo y se introduce a $21-22 \mathrm{~cm}$ en mujeres o $23-24 \mathrm{~cm}$ en hombres al nivel de la comisura labial, se debe confirmar la intubación correcta con los métodos utilizados rutinariamente (inspección, auscultación y EtCO2). (Vieira, 2017)

\section{Conclusiones}

La vía aérea difícil se ha documentado desde diferentes perspectivas a lo largo del tiempo, la misma es definida como una entidad que se presenta frecuentemente en la práctica diaria del anestesiólogo, por este motivo es importante el conocer: por qué se presenta, cómo se diagnostica, cómo se evalúa, cuál es el abordaje terapéutico, cuáles son los instrumentos o técnicas necesarias para resolverla y cuáles las posibles complicaciones que deriven de la misma. El objetivo de esta revisión es visualizar de una forma sistematizada el acercamiento que el anestesiólogo debe tener ante la vía aérea difícil; sin embargo, aún queda mucho por recorrer ya que al ser un tópico tan versátil, todos los días surge algo nuevo.

Los dispositivos supraglótigos creados y desarrollados en diferentes períodos de tiempo se encuentran dentro de los protoco- los de vía aérea difícil, son instrumentos de rescate en el manejo de la vía aérea difícil aunque no sustituyen al tubo endotraqueal. El control de la vía aérea en el paciente crítico puede resultar a menudo difícil, con un riesgo bastante elevado de su abordaje ante determinadas situaciones. Se presenta un paciente, con traumatismo raquimedular, con una vía aérea difícil en el cual el uso de los dispositivos supraglóticos (mascarilla laríngea) fue de gran utilidad en la protección de la vía aérea ante una situación de emergencia. Palabras cl

El control avanzado de la VA es una técnica habitual en el manejo de pacientes críticos. Una ventilación inadecuada del paciente va a condicionar el tratamiento y la evolución del mismo, por lo que los médicos de urgencias-emergencias deben estar preparados para tal eventualidad, disponiendo y estando familiarizados con al menos un dispositivo alternativo con el que dominar la situación. Las condiciones para el manejo de la vía aérea en la UCI deben ser lo más cercanas al ideal en cualquier circunstancia.

Lo señalado lleva a establecer la importancia de contar con el personal adecuado, el cual logre manejar lo relacionado con una colocación óptima del paciente, la iluminación conveniente, y el equipamiento necesario que permita usar alternativas de abordaje en caso de complicación. Otro factor no menos importante es la evaluación de la vía aérea antes de su abordaje. Identificar factores que impidan una correcta ventilación o intubación resulta crucial en los pacientes críticos para prevenir las complicaciones que se puedan presentar. Se han intentado aplicar las mismas medidas de evaluación de la vía aérea habitualmente empleadas en pacientes que se someten a cirugía de forma programada, pero desafortunadamente estas medidas no han podido ser evaluadas adecuadamente en pacientes que requiere intubación urgente, por lo que resulta conveniente que los intensivistas las conozcan y las apliquen cuando se

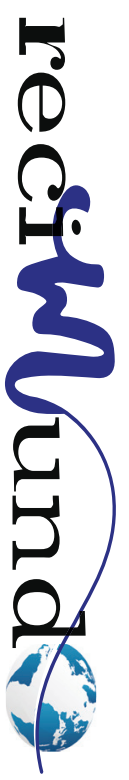


dispongan a realizar una intubación en la medida de lo posible

\section{Bibliografía}

Arias, F. (2010). Paradigmas de la Investigación Científica. España: Editorial: Luces.

Barreto, Y. (2017). Manejo de la Vía Aérea Difícil. Revista de Salud Integral de la Universidad Autónoma de México., 10-16.

Dávila, N. (2012). Paradigmas de la Investigación Científica. Pereire, Colombia: Editorial: Las Brisas.

Garza, L. (2015). Vía Aérea Difícil . Revista Chilena de Cirugía y Medicina Interna , 6-9.

La Salle, R. (2016). Técnicas de la Intubación Orotraqueal. Revista Médica de Medicina Intensivista de Costa Rica, 11-23.
Malaponte, S. (2016). Estrategias de Intubación en Vía Aérea Difícil. Revista Salud. Editorial de Cirugía Médica de Perú.,, 22-45.

Parra, F. y. (2017). Manajo y alternativas para aplicación de Vía Aérea. Revista Española de Cirugía y Anestesiología, 11-15.

Vieira, F. (2017). Técnicas y manejo de la Vía Aérea. Revista de Anestesiología de lla Universidad de la Habana en Cuba., 43., 14- 17.

\section{CITAR ESTE ARTICULO:}

Daysi Katrina, R., Quezada Miranda, E., Saltos Moran , S., \& González Mora, S. (2020). Alternativas a la intubación orotraqueal ante una vía aérea difícil. RECIMUNDO, 4(1), 26-38. doi:10.26820/recimundo/4.(1).enero.2020.26-38 